\title{
Intermittent theta-burst stimulation for upper-limb dysfunction and spasticity in spinal cord injury: a single-blind randomized feasibility study
}

\author{
Aref-Ali Gharooni ${ }^{1,2} \cdot$ Krishnan Padmakumari Sivaraman Nair $^{1} \cdot$ Debby Hawkins $^{1} \cdot$ Ian Scivill $^{1} \cdot$ Daniel Hind $^{2} \cdot$ \\ Ram Hariharan ${ }^{1}$
}

Received: 29 December 2017 / Revised: 2 April 2018 / Accepted: 5 April 2018 / Published online: 12 June 2018

(c) International Spinal Cord Society 2018

\begin{abstract}
Study design Single-blind, sham-controlled, crossover randomized feasibility study

Objectives (1) Assess the feasibility of a full-scale trial of intermittent theta-burst stimulation (iTBS) for upper-limb sensorimotor dysfunction following spinal cord injury (SCI). (2) Determine the safety and tolerability of iTBS over primary motor cortex on upper-limb function in people with spinal cord injury (SCI).

Setting Large Tertiary Spinal Injuries Centre

Methods Participants with incomplete SCI, suffering with upper-limb spasticity were recruited and randomized to receive active/sham iTBS over the hand representation of the primary motor cortex. The intervention was delivered in 10 sessions over a 2 -week period, followed by a 2 -week washout, before being crossed over to receive the alternative intervention for the same number of sessions. Feasibility was assessed by pre-specified criteria which included recruitment rate of 3 participants per month, 10 completed interventions and 10 complete data sets for 15 recruited participants with no serious adverse events. Secondary outcomes included preliminary data collection for spasticity, pain and sensorimotor function.

Results Twelve participants were recruited over 10 weeks (i.e., 4.8 per month), with 11 randomized and 10 completing the intervention protocol with no serious adverse events. Eight complete data sets were obtained as two participants failed to attend follow-up. Data from 10 participants were analyzed, with one early dropout due to an unrelated adverse event.

Conclusions It is safe and feasible to conduct a full-scale trial. Whilst iTBS has shown promising results, further research optimizing the intervention is required to improve anticipated clinical efficacy.
\end{abstract}

\section{Introduction}

Spinal cord injury (SCI) leads to necrosis and inefficient or complete loss of conduction in neural pathways. This causes impaired neurological function [1] manifesting as paralysis,

Support: International Spinal Research Trust

Electronic supplementary material The online version of this article (https://doi.org/10.1038/s41393-018-0152-5) contains supplementary material, which is available to authorized users.

Ram Hariharan

ram.hariharan@sth.nhs.uk

1 Princess Royal Spinal Injuries Centre, Sheffield Teaching Hospitals, Sheffield, UK

2 Clinical Trials Research Unit, University of Sheffield, Regent Court, 30 Regent Court, Sheffield S1 4DA, UK sensory dysfunction and other complications such as spasticity and pain [2]. These impair health-related quality of life especially in those with upper-limb functional impairments [3]. Treatments for generalized spasticity and pain have limitations due to adverse effects or limited effectiveness $[4,5]$, and there are no proven therapies to improve sensorimotor function in paralyzed muscles [6]. Therefore, it is important that new therapies are developed which can improve wellbeing in people with SCI.

Repetitive transcranial magnetic stimulation (rTMS) is a method of non-invasive brain stimulation and leads to changes in brain activity lasting beyond the stimulation period [7]. The mechanism in which rTMS alters brain activity is not fully understood, however, it has been proposed that rTMS exerts its effect via long-term potentiation (LTP) and long-term depression (LTD) like effects [7]. LTP/LTD denotes the alteration in the strength of synaptic connections based on the patterns of recent synaptic 
activity [8]. Individuals with incomplete SCI often show neurological improvements by the end of the first year postinjury [9], which occurs due to plasticity in the residual axonal connections [10]. Therefore, we hypothesize that rTMS can lead to clinical improvements in motor recovery, spasticity and pain in people with incomplete SCI due to its ability to enhance synaptic strength in residual neural pathways.

Studies investigating rTMS as a therapy for SCI have focused on sensorimotor function, spasticity and pain [11]. Despite these studies, uncertainty exists in the efficacy of rTMS for the aforementioned complications due to limited sample sizes and conflicting outcomes. Whilst previous studies have utilized high frequency rTMS protocols, no study has utilized intermittent Theta-burst stimulation (iTBS) protocol, that involves a shorter duration of patterned stimulation which has more robust effects compared to non-patterned stimulation protocols [12]. Moreover, a significant number of trials do not meet their recruitment target, leading to reduced statistical power and costly trial extensions [13]. Therefore, the primary objective of this study was to assess the feasibility of conducting a full-scale trial to investigate the efficacy of iTBS for upper limb spasticity, pain and weakness in people with incomplete cervical SCI. Secondary objectives were (1) to obtain preliminary data on the effects of iTBS on spasticity, pain and sensorimotor function, (2) to determine any adverse events and (3) to obtain feedback from the participants and patient and public involvement panel (PPI).

\section{Methods}

We conducted single-blind, sham-controlled, randomized two-period $(\mathrm{AB} / \mathrm{BA})$ crossover trial with a two-week washout period. A follow-up visit was performed at two weeks post intervention. Eligible participants were those aged between 18-70 years with incomplete cervical SCI sustained at least three-months ago, and referred to the Princess Royal Spinal Injuries Centre in Sheffield, United Kingdom. Traumatic and non-traumatic etiologies were included. Participants with cognitive abilities to give consent, no significant medical co-morbidities and spasticity affecting the upper-limbs with a combined upper-limb Modified Ashworth Score (MAS) of at least two. Exclusion criteria included ventilated individuals, normal clinical examination of upper limbs, significant upper limb contractures and joint-related limitation of movement, implanted electrical devices, pregnancy and concomitant neurological conditions including epilepsy.

Inpatients and outpatients were recruited from February to July 2016. Inpatients were approached by the clinical team and outpatients were approached directly at their

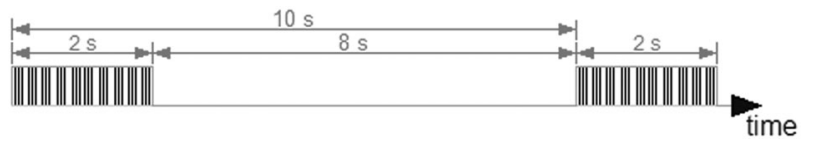

Fig. 1 iTBS waveform, illustrating a complete $2 \mathrm{~s}$ ON and $8 \mathrm{~s}$ OFF period, also includes the $2 \mathrm{~s}$ ON period of the subsequent cycle

routine appointment if the clinical team believed them to be suitable. If the inclusion criteria were met, informed written consent was obtained. In case of participants unable to sign due to hand weakness, the consent was obtained with a witness signing the consent form. Participants were randomized to the first intervention by toss of a coin and remained blind to the interventions throughout the study.

\section{Intervention}

A Magstim (Whitland, United Kingdom) SuperRapid transcranial magnetic stimulator with a $90 \mathrm{~mm}$ circular coil was used to deliver iTBS to the cortex. The coil was initially placed with its center point over the $\mathrm{Cz}$ position of the skull, which was located using the 10-20 EEG measurement system, see Fig. 1.

For this study the resting membrane threshold(RMT) was used to calculate the stimulator output energy for the delivery of the iTBS. The RMT is the stimulator energy required to first elicit a visual observation of muscle twitch in the upper limbs, during a resting state. To determine the RMT involved: a single pulse stimulation, minor coil position adjustment to selectively target the upper limb motor center in combination with gradually increasing stimulator output energy.

Active iTBS consisted of 3 stimuli at $50 \mathrm{~Hz}$ repeated at $200 \mathrm{~ms}$ intervals for $2 \mathrm{~s}$, see Fig. 1. The active iTBS used an inter-train interval of $8 \mathrm{~s}$, which was repeated 20 times for a total of 600 pulses in $200 \mathrm{~s}$. The stimulator output intensity was set to $80 \%$ of RMT which was determined at the start of each session along with final coil position [12]. Sham iTBS protocol was identical to that of active stimulation, however, the coil was rotated $90^{\circ}$ about its vertical midline axis to ensure no brain stimulation. This was delivered in 10 sessions over a period of 2 weeks, followed by a 2-week washout period, before being crossed over to receive the alternative intervention for the same number of sessions.

\section{Outcomes}

Feasibility of a full-scale future trial was assessed by prespecified feasibility criteria. The criteria were (i) recruitment rate of at least three participants per month, (ii) ten participants' complete intervention protocol, (iii) complete data for ten participants and (iv) no serious adverse events.

Secondary objectives outlined in the introduction include the suitability of the intervention protocol which was 
determined by obtaining participant feedback at a follow-up appointment regarding their experience in the trial, if they could distinguish active and sham stimulation and whether the research team could successfully implement the intervention protocol. A meeting with the spinal PPI panel at our institute was conducted to obtain further feedback. Preliminary data were obtained for spasticity, pain and sensorimotor function. Outcomes for spasticity included a combined upper-limb MAS score of bilateral elbow and wrist extension and flexion, Leeds Arm Spasticity Impact Scale (LASIS) and a Visual Analogue Scale for spasticity (VAS-S). Outcomes for sensorimotor function included the American Spinal Injuries Association (ASIA) impairment scale Upper Extremity Motor Score, Lower Extremity Motor Score, Pin Prick (PP) score and Light Touch score and the Spinal Cord Independence Measure. Pain was assessed using a Visual Analogue Scale for Pain. These were collected at baseline before the first session and after the last session in each intervention period.

\section{Sample size}

To detect the minimal clinically important difference (0.9) of a numeric rating scale (NRS) assessing spasticity [14] with a standard deviation of 2.75 at a statistical significance level of 0.05 and power of $0.80,147$ participants per group would be required using a parallel designed study or 40 participants when cross-over design efficiency is taken into account [15]. The sample in this study reflects the recruitment potential of this center within a time period, which allows us to heuristically determine whether a full-scale trial is feasible.

\section{Analytical methods}

Statistical analyses were performed using the statistical package for social sciences version 22 (IBM Corp., Armonk, N.Y. USA). Missing data were accounted for by using the last observation carried forward. Crossover trials are susceptible to the effects of participant dropout/withdrawal, therefore, participants who received the intervention in the first period, but not the second, were not included in the final analysis as they never received the intervention in the second period. Currently there is no evidence to support that iTBS has a long term effect.

Characteristics of participants include ASIA grade, level of injury, etiology, age, sex and time since injury. Feasibility outcomes were analyzed using count data, descriptive statistics and rates (e.g., recruitment rate), which was compared to the feasibility criteria and calculated sample size to assess the feasibility of a full-scale study. Feedback from participants, adverse events and the outcome of the spinal PPI meeting were reported narratively. Preliminary clinical data on spasticity, pain and sensorimotor function were reported with mean (standard deviation (SD)) and analysis of covariance was conducted to compare the efficacy (adjusted mean/intervention effect size and $95 \% \mathrm{CI}$ ) of the interventions whilst controlling for baseline values of each intervention period [16].

NHS permission and Yorkshire and the Humber ethics committee approval were obtained (Ref: 15/YH/0477) and all work was conducted in accordance with Medicines for Human Use (Clinical Trials) Regulations 2004 and subsequent amendments and ICH Good Clinical Practice (GCP). Informed consent was obtained from all participants involved in the study. The clinical trials.gov identifier is NCT02914418.

\section{Results}

\section{Participant flow}

Over a 10-week period, 25 individuals were assessed for eligibility, of which 15 (60\%) were eligible, and 3 (20\%) of those individuals declined to participate because possible adverse effects were unacceptable $(n=1)$, self-perceived spasticity was insignificant $(n=1)$ and distance required to travel was too great $(n=1)$. Ten $(40 \%)$ of individuals were ineligible with age ( $>70$ years) being the main factor $(n=$ 9 ), followed by epilepsy $(n=1)$, and additionally, the distance required to travel was mentioned $(n=4)$ as a reason for non-participation. Out of the 12 individuals consented $(48 \%), 11$ were randomized $(44 \%)$ and $1(8 \%)$ withdrew prior to randomization due to unrelated health problems. Data were analyzed for 10 participants as one (8\%) withdrew due to intolerability to iTBS. Overall, 10 participants completed the intervention protocol, and eight completed the full trial protocol as two participants were discharged from inpatient care and the distance required to travel was too great to attend the follow up session. See Fig. 2 for a flow diagram of the study.

The mean(SD) age of participants was 46.8(11.9) years with $80 \%$ of participants being male and a traumatic SCI etiology. Characteristics of individual participants are presented in Table 1.

\section{Outcomes}

Three out of four feasibility criteria were met which included 10 participants completing the intervention protocol, 12 participants recruited in 10 weeks (i.e., 4.8 participants per month) and no serious adverse events. Complete valid data were obtained from eight participants as two did not attend the follow-up visit. 
Fig. 2 Flow diagram of study

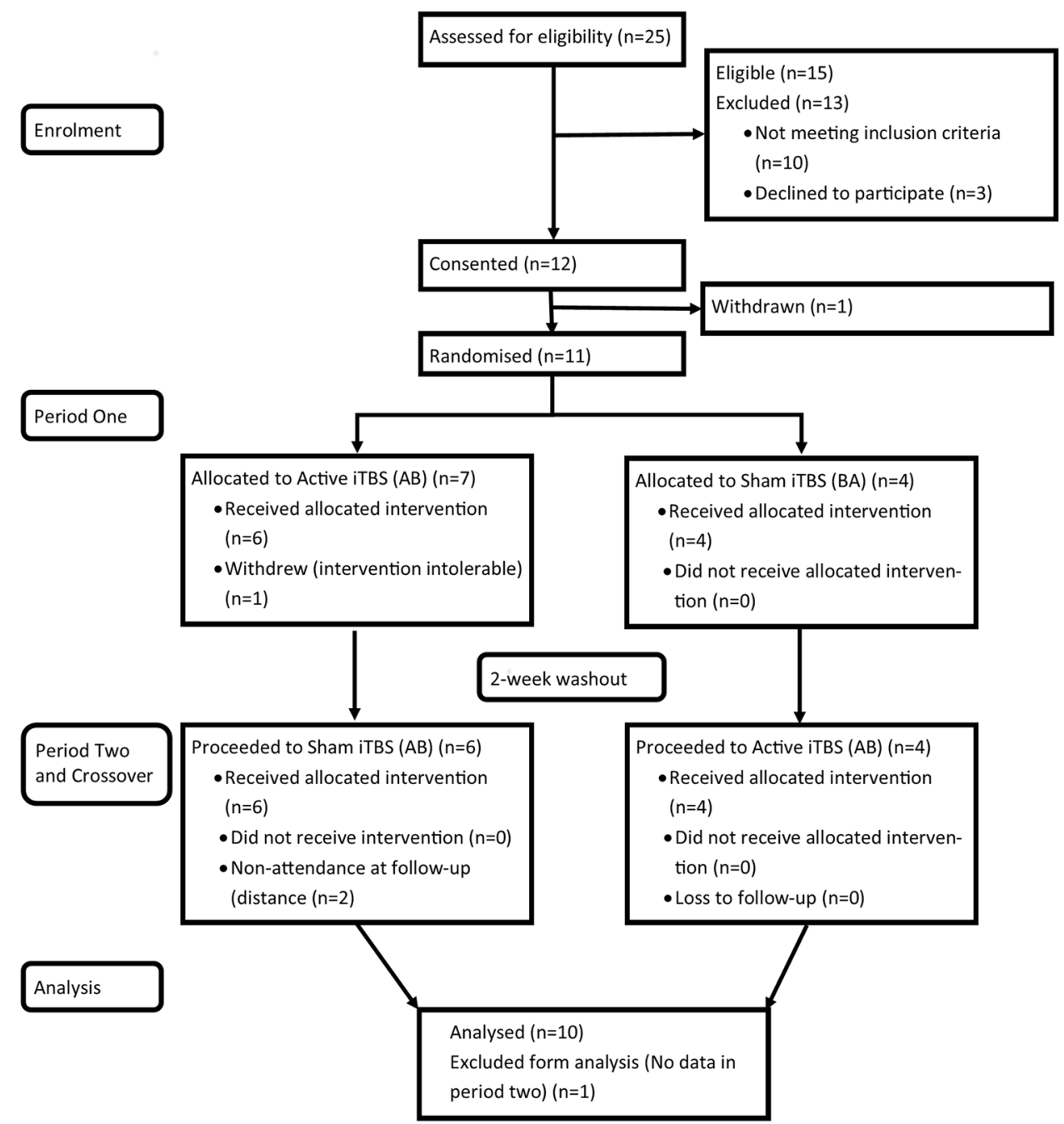

Feedback from participants was obtained at the followup visit. For all eight participants the feedback was positive with no related adverse events, and satisfactory trial documents and conduct. Five of the eight (68\%) correctly identified the order of intervention mentioning the "tapping sensation" on their head during active stimulation. Two participants couldn't identify the order of interventions and one incorrectly identified this.

Questions raised by the PPI panel included whether the washout period was long enough, minimum/maximum time post injury, number of missed sessions before being classed as a drop out, adequacy of sham stimulation, qualitative outcomes regarding participants experience and cost of caregivers for participants, travel and parking. The issues raised are all considerations for the design of any future study.

Preliminary clinical outcomes with estimates of intervention effect size and 95\% CI are displayed in Table 2. Data for individual participants are presented as supplementary information.

\section{Adverse events}

One participant reported interscapular "tightness" the morning after their first session (active) but no adverse events during or immediately after stimulation. Following discussion with the clinical team, it was decided this was unrelated to the intervention as the participant had similar experiences prior to enrollment which was attributed to ongoing spasticity and there are currently no physiological mechanisms which explain this delayed adverse event.

\section{Discussion}

The results support the feasibility in recruitment and acceptability of conducting a full-scale trial despite only three out of four criteria being met, which underlined changes to be made to the protocol. Two participants failed to attend the follow-up session due to the distance required to travel which was also cited as a reason for non- 
Table 1 Participant characteristics

\begin{tabular}{lllllllll}
\hline Participant number & AIS grade & Level of Injury & Aetiology & Age & Sex & Time since injury & Intervention order & Spinal surgery and implants \\
\hline 1 & B & C4 & NT & 32 & F & 5 months & AB & No spinal surgery \\
2 & D & C3 & T & 53 & M & 3 months & AB & No spinal surgery \\
3 & D & C3 & T & 54 & M & 3 months & AB & No spinal surgery \\
4 & C & C4 & NT & 53 & F & 4 months & AB & Surgical fixation \\
5 & C & C3 & T & 70 & M & 3 months & AB & No spinal surgery \\
6 & C & C6 & T & 35 & M & 3 months & AB & No spinal surgery \\
7 & D & C3 & T & 52 & M & 3 yr 10months & BA & Surgical fixation \\
8 & D & C3 & T & 41 & M & 2 yr 7months & BA & No spinal surgery \\
9 & C & C4 & T & 49 & M & 1 yr & BA & Surgery, no Implants \\
10 & D & C5 & T & 29 & M & 4 months & BA & Surgical fixation \\
\hline
\end{tabular}

Participant baseline characteristics

$A B$ active then sham intervention, $B A$ sham then active intervention, $T$ traumatic, $N T$ non-traumatic

Table 2 Preliminary clinical outcomes

\begin{tabular}{|c|c|c|c|c|c|c|}
\hline \multirow{2}{*}{$\begin{array}{l}\text { Outcome Measure- } \\
\text { (range) }\end{array}$} & \multicolumn{2}{|c|}{ Active rTMS $(n=10)$} & \multicolumn{2}{|c|}{ Sham rTMS $(n=10)$} & \multicolumn{2}{|c|}{ Intervention effect } \\
\hline & $\begin{array}{l}\text { Baseline-Mean } \\
\text { (SD) }\end{array}$ & $\begin{array}{l}\text { End of intervention- } \\
\text { Mean(SD) }\end{array}$ & $\begin{array}{l}\text { Baseline-Mean } \\
\text { (SD) }\end{array}$ & $\begin{array}{l}\text { End of intervention- } \\
\text { Mean (SD) }\end{array}$ & Estimate $^{a}$ & $\begin{array}{l}95 \% \text { Confidence } \\
\text { Interval }\end{array}$ \\
\hline $\begin{array}{l}\text { MAS (Combined } \\
\text { upper-limb)-(0-40) }\end{array}$ & $10.60(2.09)$ & $5.90(3.16)$ & $9.60(5.14)$ & $7.60(4.81)$ & -2.67 & -5.17 to -0.17 \\
\hline LASIS- $-(0-4)$ & $2.25(1.22)$ & $2.11(1.45)$ & $2.02(1.34)$ & $1.89(1.41)$ & 0.16 & -0.18 to 0.48 \\
\hline $\begin{array}{l}\text { VAS-S }(\mathrm{mm})- \\
(0-100)\end{array}$ & $50.80(21.85)$ & $54.90(26.20)$ & $64.50(20.67)$ & $46.70(25.76)$ & -1.99 & -21.00 to 17.01 \\
\hline UEMS- $-(0-50)$ & $28.20(13.79)$ & $31.50(14.84)$ & $28.30(14.31)$ & $31.8(14.97)$ & 0.20 & -1.90 to 2.31 \\
\hline LEMS— $(0-50)$ & $25.50(17.03)$ & $30.70(14.44)$ & $27.50(13.34)$ & $32.60(14.35)$ & -0.53 & -6.48 to 5.41 \\
\hline $\mathrm{PP}-(0-112)$ & $63.20(6.99)$ & $65.60(7.41)$ & $65.60(8.87)$ & $64.70(2.91)$ & -0.904 & -6.24 to 4.44 \\
\hline $\mathrm{LT}-(0-112)$ & $63.10(7.17)$ & $65.60(7.41)$ & $68.10(11.74)$ & $64.70(2.91)$ & 0.017 & -6.83 to 6.86 \\
\hline SCIM- $(0-100)$ & $41.50(21.61)$ & $46.80(25.81)$ & $50.10(25.26)$ & $45.40(24.03)$ & 0.405 & -4.25 to 5.06 \\
\hline $\begin{array}{l}\text { VAS-P (mm)- } \\
(0-100)\end{array}$ & $37.10(29.85)$ & $34.90(29.95)$ & $24.70(27.99)$ & $35.10(29.38)$ & -0.02 & -19.35 to 19.31 \\
\hline
\end{tabular}

Preliminary outcomes. MAS (combined upper-limb), $0-40,0=$ No increase in muscle tone, $40=$ maximum spasticity. LASIS, $0-4,0=$ No disability, $4=$ maximum disability. VAS-S, $0-100,0=$ No spasticity, $100=$ Maximum spasticity. UEMS, $0-50,0=$ total paralysis, $50=$ active movement against resistance in upper limbs. LEMS, $0-50,0=$ total paralysis, $50=$ active movement against resistance in lower limbs. PP, $0-112$, $0=$ No pin prick sensation, $112=$ Normal pin prick sensation. LT, $0-112,0=$ No light touch sensation, $112=$ Normal light touch sensation . SCIM, 0-100, $0=$ Dependant, $100=$ independent. VAS-P, $0-100,0=$ No pain, $100=$ Maximum pain

${ }^{a}$ Estimate of intervention effect adjusted for baselines as covariates

participation, therefore, multiple centers delivering the intervention would enhance recruitment and retention further improving feasibility of a future study. To address dropout and loss to follow up of $33 \%$, the sample size can be inflated accordingly, $(40 /(1-0.33)=60$ rounded $)$ requiring 60 participants in total which will require 100 persons to be screened. We believe screening 100 persons is a feasible target considering our recruitment rate.

The preliminary clinical results are worthy of further exploration Whilst we observed a reduction in upper-limb spasticity measured by MAS, this does not appear large enough to improve participants perception of spasticity or improve their functionality as measured by VAS-S and
LASIS. These outcomes are concordant with previous findings [11]. Effects on sensorimotor function and pain indicate that the intervention protocol is unlikely to lead to any significant improvements in either of these outcomes. These findings concordant with some previous studies [17] however, a major limitation is that participants were not recruited based on their level or type of pain. As iTBS has shown some tendency to improve spasticity, we believe further studies are warranted to further develop optimized neuromodulatory protocols.

This was the first trial utilizing iTBS in SCI, however, there are a number of limitations. MAS has been recommended as an outcome measure for spasticity despite poor 
inter-rater reliability and correlation with patient reported outcome measures [18]. The VAS is limited by low testretest reliability [19] however, an alternative is the NRS which has superior test-retest reliability [14]. This trial also lacked neurophysiological assessments of spasticity which can provide a more objective outcome [20]. Determining RMT by visual observation of a twitch is reliable [21], however, it can overestimate the threshold when compared to electromyographic determination [22]. Furthermore, the effects of iTBS on motor-evoked potentials (MEPs) in healthy participants shows variability and further research is required to determine whether this correlates with motor behavior [12, 23]. Almost two-thirds of participants attending follow-up correctly identified the order of interventions, which highlights inadequate sham stimulation. It could be improved by placing surface electrodes on the participants head to mimic the tapping sensation during iTBS which has been conducted previously [24]. A further source of bias was that outcome assessors were not blind to the intervention participants received. In addition, preliminary data of clinical outcomes should be interpreted with caution due to the small sample size. This study also highlights the increasing age of people with SCI as $90 \%$ of ineligibility was due to advanced age, which may pose problems for interventions enhancing neuroplasticity as this reduces during the ageing process [25]. This was a single center feasibility study therefore, future multicenter trials may require further studies to determine feasibility across multiple sites.

\section{Conclusion}

In conclusion, iTBS is a safe and acceptable intervention for upper-limb sensorimotor dysfunction in people with SCI. It is feasible to conduct a larger study, however, modifications to the protocol are required to enhance recruitment and retention. Whilst iTBS has shown promising results to reduce upper-limb spasticity, further research in optimizing the intervention protocol is required to improve anticipated efficacy.

\section{Acknowledgements}

Author contributions AAG was responsible for conception and design of study, acquisition of data, analysis, interpretation, writing the report and final approval. RH was responsible for design of study, acquisition of data, interpretation and final approval. KPSN was responsible for design of study, analysis, interpretation and final approval. DH was responsible for data management, study interpretation and final approval. DH was responsible for design of study, study interpretation and final approval. IS was responsible for technical guidance and support, interpretation and final approval.

Funding This trial was funded by the International Spinal Research Trust. The funder had no role in the design, conduct, analysis or writing of the report.

\section{Compliance with ethical standards}

Conflict of interest The authors declare that they have no conflict of interest.

\section{References}

1. James ND, Bartus K, Grist J, Bennett DLH, McMahon SB, Bradbury EJ. Conduction failure following spinal cord injury: functional and anatomical changes from acute to chronic stages. $\mathrm{J}$ Neurosci. 2011;31:18543-55.

2. Sezer N, Akkus S, Ugurlu FG. Chronic complications of spinal cord injury. World J Orthop. 2015;6:24-33.

3. Craven C, Hitzig SL, Mittmann N. Impact of impairment and secondary health conditions on health preference among Canadians with chronic spinal cord injury. J Spinal Cord Med. 2012;35:361-70.

4. Taricco M, Pagliacci MC, Telaro E, Adone R. Pharmacological interventions for spasticity following spinal cord injury: results of a Cochrane systematic review. Eura Med. 2006;42:5-15.

5. Mehta S, McIntyre A, Janzen S, Loh E, Teasell R. Systematic review of pharmacologic treatments of pain after spinal cord injury: an update. Arch Phys Med Rehabil. 2016;97: 1381-1391.e1.

6. Harvey LA. Physiotherapy rehabilitation for people with spinal cord injuries. J Physiother. 2016;62:4-11.

7. Hoogendam JM, Ramakers GM, Di Lazzaro V. Physiology of repetitive transcranial magnetic stimulation of the human brain. Brain Stimul. 2010;3:95-118.

8. Bliss TVP, Cooke SF. Long-term potentiation and long-term depression: a clinical perspective. Clinics. 2011;66:(Suppl 1)3-17.

9. Scivoletto G, Tamburella F, Laurenza L, Molinari M. Distribution-based estimates of clinically significant changes in the International Standards for Neurological Classification of Spinal Cord Injury motor and sensory scores. Eur J Phys Rehabil Med. 2013;49:373-84.

10. Martin JH. Harnessing neural activity to promote repair of the damaged corticospinal system after spinal cord injury. Neural Regen Res. 2016;11:1389-91.

11. Awad BI, Carmody MA, Zhang X, Lin VW, Steinmetz MP. Transcranial magnetic stimulation after spinal cord injury. World Neurosurg. 2015;83:232-5.

12. Huang YZ, Edwards MJ, Rounis E, Bhatia KP, Rothwell JC. Theta burst stimulation of the human motor cortex. Neuron. 2005;45:201-6.

13. Campbell MK, Snowdon C, Francis D, Elbourne D, McDonald AM, Knight R, et al. Recruitment to randomised trials: strategies for trial enrollment and participation study. The STEPS study. Health Technol Assess. 2007;11:iii, ix-105.

14. Farrar JT, Troxel AB, Stott C, Duncombe P, Jensen MP. Validity, reliability, and clinical importance of change in a 0-10 numeric rating scale measure of spasticity: a post hoc analysis of a randomized, double-blind, placebo-controlled trial. Clin Ther. 2008;30:974-85.

15. Wellek S, Blettner M. On the proper use of the crossover design in clinical trials: part 18 of a series on evaluation of scientific publications. Dtsch Arztebl Int. 2012;109:276-81.

16. Mehrotra DV. A recommended analysis for $2 \times 2$ crossover trials with baseline measurements. Pharm Stat. 2014;13:376-87.

17. Jette F, Cote I, Meziane HB, Mercier C. Effect of single-session repetitive transcranial magnetic stimulation applied over the hand versus leg motor area on pain after spinal cord injury. Neurorehabil Neural Repair. 2013;27:636-43.

18. Steeves JD, Lammertse D, Curt A, Fawcett JW, Tuszynski MH, Ditunno JF, et al. Guidelines for the conduct of clinical trials for 
spinal cord injury (SCI) as developed by the ICCP panel: clinical trial outcome measures. Spinal Cord. 2006;45:206-21.

19. Brunelli C, Zecca E, Martini C, Campa T, Fagnoni E, Bagnasco $\mathrm{M}$, et al. Comparison of numerical and verbal rating scales to measure pain exacerbations in patients with chronic cancer pain. Health Qual Life Outcomes. 2010;8:1-8.

20. Voerman GE, Gregoric M, Hermens HJ. Neurophysiological methods for the assessment of spasticity: the Hoffmann reflex, the tendon reflex, and the stretch reflex. Disabil Rehabil. 2005;27:33-68.

21. Varnava A, Stokes MG, Chambers CD. Reliability of the "observation of movement" method for determining motor threshold using transcranial magnetic stimulation. J Neurosci Methods. 2011;201:327-32.
22. Westin GG, Bassi BD, Lisanby SH, Luber B. Determination of motor threshold using visual observation overestimates transcranial magnetic stimulation dosage: Safety implications. Clin Neurophysiol. 2014;125:142-7.

23. Hinder MR, Goss EL, Fujiyama H, Canty AJ, Garry MI, Rodger J, et al. Inter- and Intra-individual variability following intermittent theta burst stimulation: implications for rehabilitation and recovery. Brain Stimul. 2014;7:365-71.

24. Gomes-Osman J, Field-Fote EC. Improvements in hand function in adults with chronic tetraplegia following a multiday $10-\mathrm{Hz}$ repetitive transcranial magnetic stimulation intervention combined with repetitive task practice. J Neurol Phys Ther. 2015;39:23-30.

25. Burke SN, Barnes CA. Neural plasticity in the ageing brain. Nat Rev Neurosci. 2006;7:30-40. 